\title{
Non-invasive assessment of early and acute myocarditis in a rat model using cardiac magnetic resonance tissue tracking analysis of myocardial strain
}

\author{
Jing Zhu, Yushu Chen, Ziqian Xu, Shiyu Wang, Lei Wang, Xiaoxin Liu, Fabao Gao \\ Department of Radiology, West China Hospital, Sichuan University, Chengdu, China \\ Correspondence to: Fabao Gao. Department of Radiology, West China Hospital, Sichuan University, 37 Guoxue Road, Chengdu 610041 China. \\ Email: gaofabao@wchscu.cn.
}

\begin{abstract}
Background: Myocardial strain analysis can provide diagnostic and prognostic information for myocarditis. The aim of the present study was to assess early and acute myocarditis in a rat model using cardiac magnetic resonance tissue tracking (CMR-TT) for myocardial strain analysis. We compared the strain's findings with the histological and immunohistochemical results.

Methods: Experimental autoimmune myocarditis (EAM) was induced by footpad injections of porcine cardiac myosin. The rats were examined by 7.0 T preclinical CMR at day $14(\mathrm{n}=15)$ and day $21(\mathrm{n}=16)$ after EAM induction and the two control groups (each $\mathrm{n}=15$ ) were also examined at day 14 and day 21, respectively. Using CMR-TT, we found a global peak systolic radial strain $\left(\operatorname{Err}_{\mathrm{SAX}}\right)$ and a circumferential strain $\left(\mathrm{Ecc}_{\mathrm{SAX}}\right)$ from the short-axis cine views and a radial strain $\left(\operatorname{Err}_{\mathrm{LAX}}\right)$ and a longitudinal strain $\left(\mathrm{Ell}_{\mathrm{LAX}}\right)$ from the long-axis cine views, which were calculated by dedicated TT software. Subsequently, histological and immunohistochemical evaluations were performed.

Results: $\mathrm{Ell}_{\mathrm{LAX}}$ significantly decreased in early myocarditis compared with the control $(-23.40 \% \pm 1.48 \%$ vs. $-22.02 \% \pm 0.81 \%, \mathrm{P}<0.05)$. $\operatorname{Err}_{\mathrm{SAX}}, \mathrm{Ecc}_{\mathrm{SAX}}, \mathrm{Err}_{\mathrm{LAX}}$, and $\mathrm{El}_{\mathrm{LAX}}$ values significantly reduced in acute myocarditis compared with the controls $\left(\operatorname{Err}_{\mathrm{SAX}}: 34.27 \% \pm 9.80 \%\right.$ vs. $49.76 \% \pm 4.97 \%, \mathrm{Ecc}_{\mathrm{SAX}}:-18.98 \% \pm 3.69 \%$ vs. $-24.13 \pm 1.23, \operatorname{Err}_{\mathrm{LAX}}: 33.21 \% \pm 10.24 \%$ vs. $49.59 \% \pm 5.69 \%$, and $\mathrm{Ell}_{\mathrm{LAX}}:-17.75 \% \pm 3.58 \%$ vs. $-23.39 \% \pm 1.48 \%$; $\mathrm{P}<0.001$, respectively). The receiver operating characteristic curve showed that myocardial strain analysis had a good diagnostic performance in early and acute myocarditis. The pathological evaluation revealed that inflammatory lesions began to appear in early myocarditis and peaked in acute myocarditis.

Conclusions: The CMR-TT strain analysis allowed accurate and reliable evaluation of early and acute myocarditis in a rat model, and has the potential to serve as a diagnostic indicator for the assessment of myocardial dysfunction in myocarditis.
\end{abstract}

Keywords: Cardiac magnetic resonance; tissue tracking; experimental autoimmune myocarditis; myocardial strain analysis; myocarditis

Submitted Jan 15, 2020. Accepted for publication Jul 23, 2020.

doi: 10.21037/qims-20-122

View this article at: http://dx.doi.org/10.21037/qims-20-122

\section{Introduction}

Myocarditis is a non-ischemic inflammatory disease characterized by inflammatory cellular infiltration and accompanied by degenerative and/or necrosis of cardiomyocytes (1), is an important cause of dilated cardiomyopathy, and has a poor prognosis (2-5). Heterogeneous clinical manifestations, ranging from asymptomatic to non-specific symptoms (i.e., arrhythmia, acute heart failure, infarct-like representations, sudden cardiac death, and subsequent cardiac dysfunction), lead to 
challenges and difficulties with acute myocarditis diagnosis $(3,4,6)$. Missed diagnosis or misdiagnosis may increase the probability of adverse outcomes, such as chronic heart failure. Therefore, a well-timed and accurate diagnosis is vital for tailored intervention to improve symptoms and prognosis. Endomyocardial biopsy (EMB) is widely accepted as the diagnostic golden standard. Despite improved safety and a reduction in sample errors $(7,8)$, the widespread use of EMB is restricted because the immunohistological and molecular analyses involved in it call for specialized expertise and equipment that are beyond the reach of most medical institutions and hospitals (9).

Cardiovascular magnetic resonance (CMR) has been increasingly used for the non-invasive assessment of myocarditis due to its good diagnostic and prognostic values (10). Indeed, the topographic distribution of late gadolinium enhancement (LGE) and histological inflammation were matched in a rat model of acute myocarditis, as confirmed by CMR (11). Although Lake Louise criteria (LLC) for the CMR-based diagnosis of acute myocarditis have been used in clinical practice and research (2), it has some shortcomings, such as low diagnostic accuracy (12), especially in diffuse lesions. The updated LLC have the potential to improve the diagnostic value, but still need to be confirmed and standardized (13). Cardiac dysfunction is considered a feature of myocardial inflammation and is a supportive diagnostic criteria for myocarditis (13). It has recently been demonstrated that tissue tracking (TT), one of the latest quantitative CMR strain analysis techniques, can rapidly and reliably evaluate subtle cardiac dysfunction (14). Strain analysis can reflect global and regional left ventricular functional impairment in longitudinal, circumferential, and radial directions, even without visually abnormal wall motion, with a high signal-to-noise ratio and low investment of time (15) Strain analysis is more sensitive and can offer more comprehensive information compared with left ventricular ejection fraction (16-18). Several studies have reported deformation abnormality and shown that strain analysis has the potential to provide additional diagnostic information in acute myocarditis $(19,20)$. However, in these studies, no biopsy was acquired to confirm myocarditis, which could have confounded the study cohort, while early myocarditis is rarely studied due to its mild symptoms and difficult diagnosis.

As an established animal model manifesting human myocarditis in different stages $(21,22)$ and based on its histological characterization resembling human myocarditis $(21,23)$, experimental autoimmune myocarditis (EAM) could further facilitate understanding of the disorder and might have the potential to serve as an indicator for testing and developing therapies (24). We aimed to investigate the changes of myocardial strain in early and acute myocarditis. Using an EAM rat model, the present study was intended to assess whether strain analysis could discriminate early myocarditis from the healthy myocardium, and to further define the diagnostic role of strain parameters in acute myocarditis.

\section{Methods}

\section{Animals}

Sixty-one male Lewis rats weighing $200 \mathrm{~g}$ were used in the present study. The animals were randomized into four groups: the early control group $(n=15)$, early myocarditis group $(\mathrm{n}=15)$, acute control $(\mathrm{n}=15)$ group, and acute myocarditis group $(\mathrm{n}=16)$. They were kept under standard conditions with a mean temperature of $22 \pm 2{ }^{\circ} \mathrm{C}$, a mean relative humidity of $55 \% \pm 10 \%$, and a defined day-andnight-rhythm of 12-hour light and 12-hour dark. The study was approved by the Institutional Animal Care and Use Committee of the Animal Experiment Center, West China Hospital, Sichuan University, Chengdu, Sichuan, China.

\section{EAM induction}

EAM induction was performed in accordance with a previous study (21). Briefly, porcine cardiac myosin (antigen; PCM M0531; Sigma) was emulsified with equal volume of complete Freund's adjuvant (CFA; F5881; Sigma). On days 0 and 7 , each rat of the experimental group was subcutaneously injected with $0.2 \mathrm{~mL}$ emulsion in the footpad, containing $1 \mathrm{mg}$ PCM. The control rats were injected with only $0.1 \mathrm{~mL}$ CFA on the same days.

\section{CMR protocol}

On days 14 and 21, CMR acquisition was performed using a 7.0 T preclinical system (Bruker BioSpec 70/30; Ettlingen) equipped with a dedicated cardiac coil for rats. The animals were placed in the coil headfirst in the supine position, with electrodes placed on all four limbs for monitoring electrocardiography (ECG). A pressure transducer was placed under the abdomen for respiratory gating. The anesthesia was maintained with isoflurane (1-2\% isoflurane, oxygen flow rate: $1 \mathrm{~L} / \mathrm{min}$ ). The CMR scan was performed 
after the heart rate $(390 \pm 20 \mathrm{bpm})$ and respiratory rate $(35 \pm 5$ bpm) were stabilized.

Slice levels were adjusted according to the individual heart axis. Images of three horizontal long-axis (2,3,4-chamber view) stacks and a short-axis stack covering the left ventricle (LV) from the base to the apex were scanned by a cardiac FLASH cine sequence. Imaging parameters were the following: repetition time/echo time (TR/TE), 8.0/2.5 ms; FA, $15^{\circ}$; slice thickness, $1.5 \mathrm{~mm}$; interslice spacing, $0 \mathrm{~mm}$; field of view (FOV), $50 \times 50 \mathrm{~mm}$; matrix size, 256 $\times 256$; read resolution, $0.26 \times 0.26 \mathrm{~mm} /$ pixel; and number of signal averages, 2. LGE, based on the FLASH cine sequence, was performed at the same slice locations as the cine images. LGE imaging was performed 7-10 minutes after the administration of $1 \mathrm{mmol} / \mathrm{kg}$ gadolinium-diethylenetriaminepentaacetic acid. Imaging parameters were the same as those of the abovementioned cine sequence.

\section{Cardiac function and strain analysis}

All the CMR analyses were performed using commercially available software (cvi42 version 5; Circle Cardiovascular Imaging). Standard CMR parameters, including left ventricular end-diastolic volume, left ventricular endsystolic volume, left ventricular mass, stroke volume, and left ventricular EF were derived from a short-axis cine stack by semiautomated endocardial and epicardial contour tracing.

Horizontal long-axis cine images were tracked to derive longitudinal global strain, and short-axis cine images were used to derive radial and circumferential strains. Tissue tracking analysis was performed by manually drawing the endocardial and epicardial border in the end-diastolic phase on a short-axis and 2-, 3-, 4-chamber long-axis views, and then automatically tracked throughout the cardiac cycle. Strain parameters included global peak radial strain from the short axis $\left(\operatorname{Err}_{\mathrm{SAX}}\right)$ and long axis $\left(\operatorname{Err}_{\mathrm{LAX}}\right)$, global peak circumferential strain $\left(\mathrm{Ecc}_{\mathrm{SAX}}\right)$ from the short axis, and global peak longitudinal strain ( $\mathrm{Ell}_{\mathrm{LAX}}$ ) from the long axis.

\section{LGE analysis}

The presence or absence of LGE was visually and qualitatively determined, and the amount of LGE was quantified based on short-axis images to assess the extent of enhancement by manually tracing endocardial and epicardial contours. Positive enhancement area was defined as voxels with a signal enhancement $>5$ standard deviations (SD) of the signal intensity of the normal myocardium (as defined by a manually placed region of interest), while excluding any visible artifacts $(10,25,26)$. The percentage of the enhanced myocardium was calculated.

\section{Morphometric and histopathological analysis}

All the rat hearts were explanted by thoracotomy after CMR. Macroscopy was evaluated according to the macroscopic morphology and pericardial effusion in previous studies (Macroscopic morphology: 0, no inflammatory alteration; 1 , focal inflammatory signs; 2 , multiple/diffuse inflammatory signs one-third or less of heart size; and 3, diffuse inflammatory signs greater than one-third of heart size. Pericardial effusion: 0 , no effusion; 1 , mild effusion; 2, massive effusion) (11,27).

After formalin fixation (4\%) for 6 hours, the hearts were dehydrated. For paraffin embedding and sectioning, 2-mm-thick slices were prepared from the formalinfixed myocardium. On the day following the sectioning, the tissue was effused in paraffin and stored at $21^{\circ} \mathrm{C}$. A microtome was then used to afford 5 - $\mu$ m-thick slices, which were transferred into warm water and placed on a slide. Hematoxylin and eosin staining and Masson trichrome staining were employed to observe inflammation, necrosis, and fibrosis.

The severity of myocarditis was histologically analyzed according to the following histological criteria (27): density of inflammatory infiltration (graded 0-3), defined by the presence of leucocytes; strength of inflammatory destruction with rarefaction and pushing apart of heart muscle fibers (graded 0-3); occurrence of pericarditis (graded 0-2); and the occurrence of necrosis (graded 0-3). The results were input into a microscopic scoring system, with a maximum score of 11 points, to express the severity of myocarditis (11). Image analysis software (ImageJ) was used to calculate collagen volume fraction $(\mathrm{CVF}$; CVF $=$ myocardial collagen fiber area/total area of the image). CVF was randomly counted in five random microscopic fields at $\times 20$ magnification under a microscope, and the average value was taken.

\section{Immunobistochemical investigation}

To further evaluate the inflammatory response in the myocardium, immunohistochemical examination was performed to study the infiltration of inflammatory cells 
in cardiac tissues. Briefly, the cardiac tissues were fixed with $4 \%$ paraformaldehyde, embedded in paraffin blocks, and then cut into 5- $\mu \mathrm{m}$-thick sections. Anti-CD68 (1:500) antibodies were applied to acetone-fixed cryosections. Cells were counted in 10 random microscopic fields at $\times 20$ magnification under a microscope. Image $\mathrm{J}$ was used to calculate the ratio of positive to all area, and the average value was taken.

\section{Statistical analysis}

Statistical analysis was performed using MedCalc 5 (Mariakerke), Prism 5 (GraphPad Software), and SPSS version 24.0 (IBM). Continuous variables are expressed as mean \pm SD for normally distributed parameters, and as median and interquartile ranges for non-normally distributed variables. Categorical variables are presented as percentages. Intergroup comparison was assessed by independent samples $t$-test. Receiver operating characteristic (ROC) curve analysis was used to assess the diagnostic performance of strain parameters and to determine the optimal sensitivity and specificity of various cut-off values for the discrimination of myocarditis. The diagnostic performance of strain parameters was compared using the area under the curve (AUC). $\mathrm{P}<0.05$ was defined as statistically significant.

\section{Results}

On day 14 , there were no significantly abnormal changes in the heart structure and size, but there were a few white infiltration spots on the immunized rats. On day 21, all the immunized rats developed significant enlargement of the myocardium and markedly increased biventricular wall thickness. The cardiac surface was covered with many white infiltration spots, 14 of which had pericardial effusion. No abnormality was found in the heart of the two control groups. The median macroscopic score was 1 (range: $0-1$ ) in the EAM group on day 14, 5 (range: 2.5-5) in the EAM group on day 21 , and 0 (range: 0 ) in the two control groups,

All the EAM rats met the histological criteria of myocarditis. Fourteen days after immunization, microscopic examination showed foci of inflammatory cell infiltration and foci of myocardial tissue necrosis along with mild myocardial interstitial edema. On day 21 , we noted a significant increase in inflammatory infiltration and myocardial necrosis, which was accompanied by fibrosis of different degrees. The control groups had a histologically normal myocardium. The median microscopic score was 1 (range: 1-2) in the EAM group on day 14, 9 (range: 6-10.75) in the EAM group on day 21, and 0 (range: 0 ) in the control groups. CVF increased in the EAM group on day 14 , but there was no statistical difference compared with the control group $(2.71 \% \pm 0.35 \%$ vs. $2.54 \% \pm 0.16 \%$, $\mathrm{P}>0.05)$. CVF was significantly higher in the immunized rats on day $21(16.53 \% \pm 3.12 \%$ vs. $2.31 \% \pm 0.20 \% ; \mathrm{P}<0.001)$ than that in the control group rats. Myocardial lesions were predominately located in the epicardial and mid-wall of the $\mathrm{LV}$ wall (Figure 1). CD68 was increased in the EAM group on day $14(7.79 \% \pm 1.48 \%$ vs. $1.73 \% \pm 0.17 \% ; \mathrm{P}<0.001)$ and day $21(39.95 \% \pm 2.79 \%$ vs. $1.64 \% \pm 0.31 \%, \mathrm{P}<0.001)$ when compared with the control group (Figure 1).

Increased regional wall thickness and pericardial effusion were observed in the EAM group on day 21 (Figures 2 and 3), whereas these abnormal changes were not found in the EAM group or the two control groups on day 14. On day 14 , no statistical difference in conventional LV functional and morphological parameters were discovered between the immunized groups and the control groups. On day 21, a significant difference was discovered between the two groups, with the exception of end-systolic volume $(\mathrm{P}<0.05)$ (Table 1).

LV strain analysis revealed that only E $11_{\mathrm{LAX}}$ $(-23.05 \% \pm 1.54 \%$ vs. $-21.96 \% \pm 0.99 \% ; \mathrm{P}<0.05)$ was statistically different between the EAM groups and the control groups on day 14. On day 21, all the strain indices were significantly lower in the EAM groups than in the control groups $\left(\operatorname{Err}_{\mathrm{SAX}}: 34.27 \% \pm 9.80 \%\right.$ vs. $48.33 \% \pm 5.86 \%$; $\mathrm{Ecc}_{\mathrm{SAX}}$ : $-18.98 \% \pm 3.69 \%$ vs. $-23.67 \% \pm 1.57 \%$; $\mathrm{Err}_{\mathrm{LAX}}: 33.21 \% \pm 10.24 \%$ vs. $48.48 \% \pm 6.50 \%$; $\mathrm{Ell}_{\mathrm{LAX}}$ : $-17.75 \% \pm 3.58 \%$ vs. $-23.18 \% \pm 1.74 \%$. All $\mathrm{P}<0.001$ ) (Table 2).

Qualitative analysis of LGE demonstrated that no positive LGE was visible in EAM rats on day 14, whereas positive LGE was visible in nine immunized rats and was mainly located in the epicardial and mid-walls of the LV, corresponding to the histopathological distribution. LGE quantification revealed that the extent of enhancement was $36.35 \% \pm 19.18 \%$ (Figure 3).

ROC analysis assessed the sensitivity and specificity of strain parameters according to the histological finding (Figure 4). On day 14, only Ell $\mathrm{LAX}_{\text {had }}$ a diagnostic value indicating myocardial impairment. On day 21 , all the global strain parameters displayed an excellent diagnostic value for detecting cardiac function involvement (all AUC $>0.8$, $\mathrm{P}<0.0001)$. The diagnostic value of $\mathrm{Ell}_{\mathrm{LAX}}$ was the highest, with a cutoff value of $-21.12 \%$, resulting in a sensitivity, 

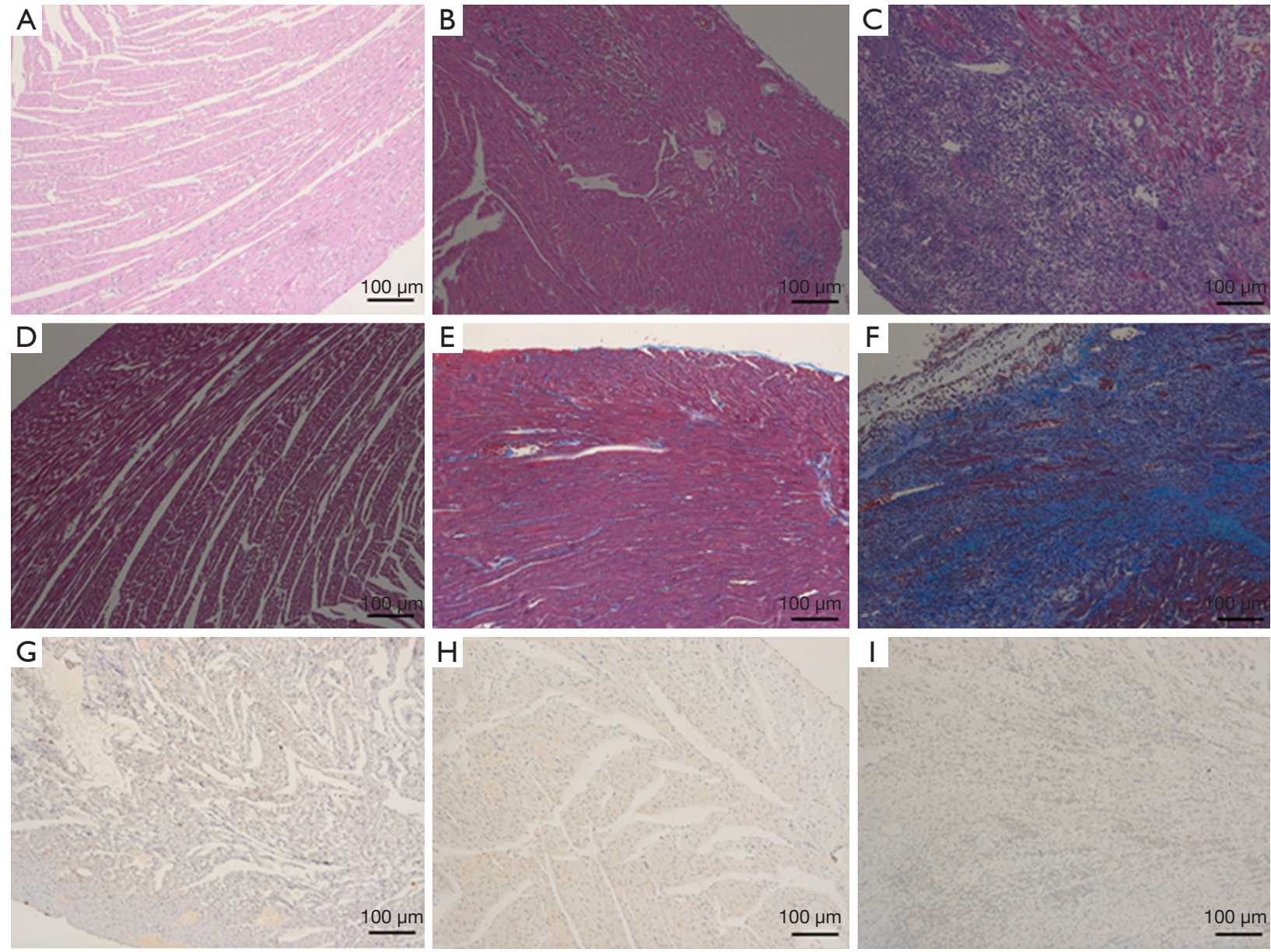

Figure 1 Myocardial tissue sections in the three groups. Images show histological (A, B, C: hematoxylin and eosin staining; D, E, F: Masson staining) and immunohistochemical (G, H, I: CD68 staining) findings in the control groups, early myocarditis group, and acute myocarditis group.

specificity, positive predictive value, and negative predictive value of $88,100,100$, and 88 , respectively; however, its AUC was similar to the AUC of the other three global strain parameters (Table 3).

\section{Discussion}

In the present study, we evaluated CMR-TT-derived strain changes in an early and acute myocarditis model of rats, and compared these strain parameters with histological and immunohistochemical results. The main findings of the study were that myocardial strain analysis can sensitively detect early cardiac function impairment in early myocarditis, all the global strain parameters showed excellent diagnostic value for the detection of acute myocarditis, and myocardial strain analysis was found to reliably assess EAM at different time points.

In all of the global strain parameters, including $\operatorname{Err}_{\mathrm{SAX}}$, $\mathrm{Ecc}_{\mathrm{SAX}}, \mathrm{Err}_{\mathrm{LAX}}$, and $\mathrm{Ell}_{\mathrm{LAX}}$, only the longitudinal strain was statistically significant between the early myocarditis group and control group, whereas all strain parameters showed statistical differences between the acute myocarditis group and the control group, indicating that myocardial strain analysis can not only detect early cardiac dysfunction in early myocarditis but facilitate a comprehensive evaluation of impairment of cardiac function in acute myocarditis. Studies on early myocarditis are scarce, and to the best of our knowledge, our study was the first to use strain analysis to evaluate early myocarditis. In the rat model of early myocarditis, we found that $\mathrm{El}_{\mathrm{LAX}}$ decreased in EAM rats with normal EF when compared with the control, suggesting that $\mathrm{Ell}_{\mathrm{LAX}}$ could detect subtle impairment of 
AHA segmentation

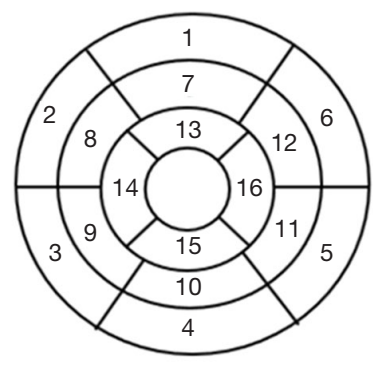

Control

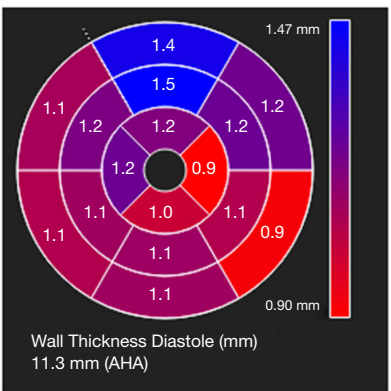

Control $(n=15)$

Early myocarditis $(n=15)$

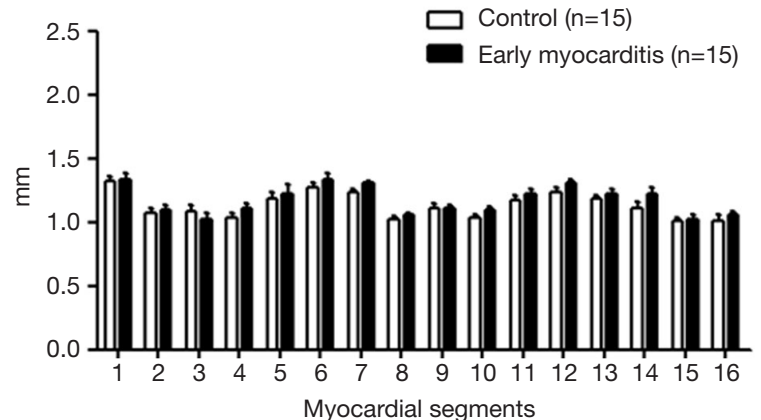

Early myocarditis
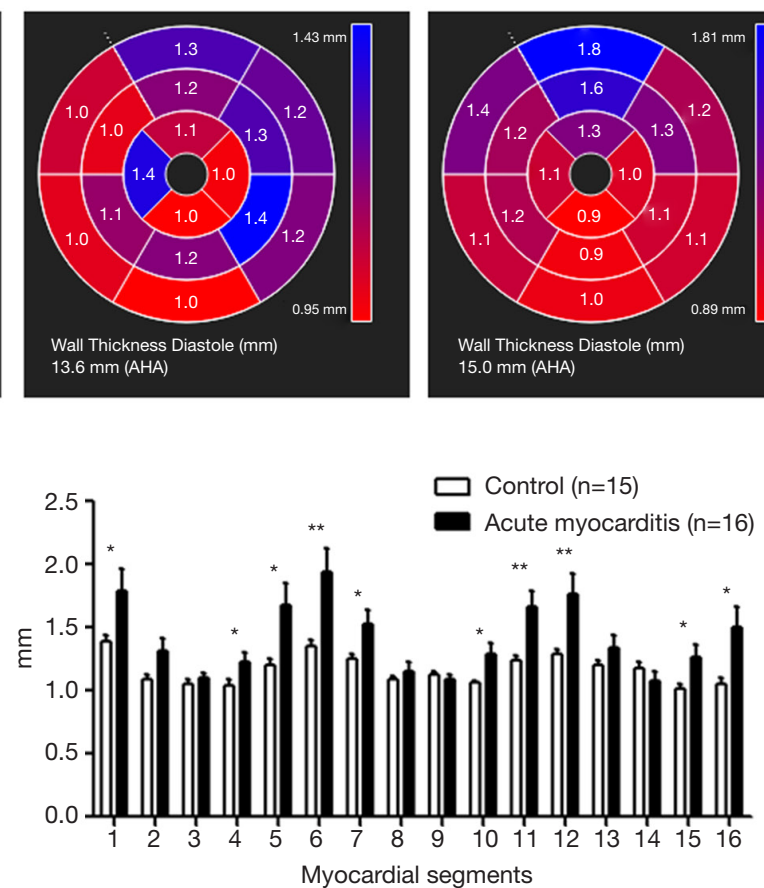

Figure 2 Left ventricle wall thickness of the myocardial segments in rats by cardiac magnetic resonance. The upper left represented the 17 myocardial segment division according to the American Heart Association (AHA). Representative 16-segment model showed increased diastolic wall thickness in acute myocarditis (upper row). There were no significant increases in segmental wall thickness in early myocarditis, whereas there was a significant increase in acute myocarditis (lower row). ${ }^{*} \mathrm{P}<0.05,{ }^{* *} \mathrm{P}<0.01$.
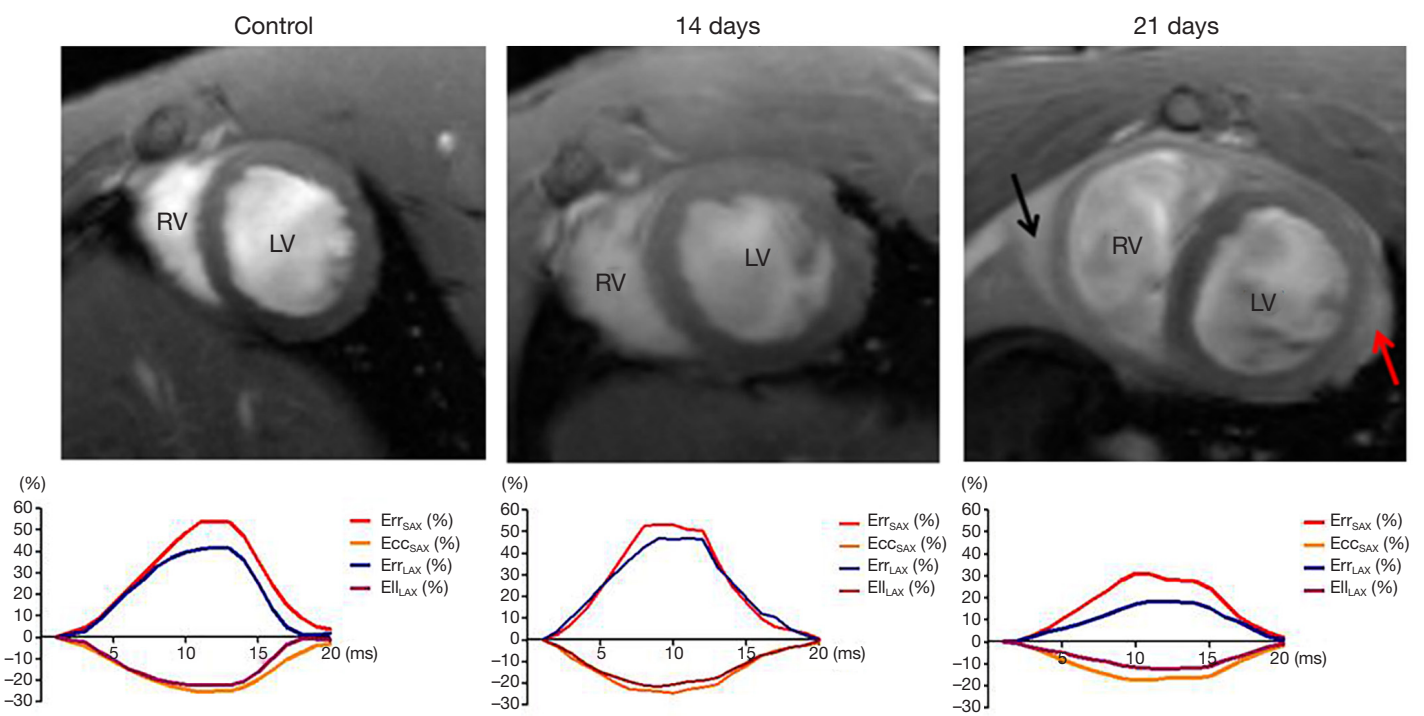

Figure 3 Late gadolinium enhancement (LGE) images and strain curves of Control and early myocarditis (14 days) and acute myocarditis (21 days). LGE images showed no visible intensive foci in the Control group and early myocarditis group, whereas there were typical patchy epicardial and mid-myocardial inflammatory/necrotic lesions in the acute myocarditis group (hyperintense, red arrow; pericardial effusion, black arrow). Strain time curves in a cardiac cycle showed that strain was reduced in acute myocarditis. $\mathrm{Ecc}_{\mathrm{SAX}}$, circumferential strain; $\mathrm{Ell}_{\mathrm{LAX}}$, longitudinal strain; $\mathrm{Err}_{\mathrm{LAX}}$, radial strain from long axis; Err $\mathrm{SAX}_{\mathrm{X}}$, radial strain from short axis; LV, left ventricle; RV, right ventricle. 
Table 1 Comparison of functional and morphological parameters in the EAM group and control group

\begin{tabular}{lcccc}
\hline \multirow{2}{*}{ Parameters } & \multicolumn{2}{c}{ Day 14} & & \multicolumn{2}{c}{ Day 21} \\
\cline { 2 - 5 } \cline { 3 - 5 } & EAM group & Control group & EAM group & $67.00 \pm 3.60$ \\
EF $(\%)$ & $64.65 \pm 5.35$ & $66.40 \pm 3.20$ & $59.14 \pm 5.90^{* * *}$ & $0.27 \pm 0.02$ \\
SV $(\mathrm{mL})$ & $0.22 \pm 0.04$ & $0.23 \pm 0.02$ & $0.18 \pm 0.04^{* * *}$ & $0.32 \pm 0.05$ \\
LV mass $(\mathrm{g})$ & $0.29 \pm 0.03$ & $0.30 \pm 0.03$ & $0.42 \pm 0.13^{* *}$ & $0.37 \pm 0.02$ \\
EDV $(\mathrm{mL})$ & $0.34 \pm 0.05$ & $0.35 \pm 0.09$ & $0.31 \pm 0.06^{* * *}$ & $0.14 \pm 0.01$ \\
ESV $(\mathrm{mL})$ & $0.13 \pm 0.03$ & $0.13 \pm 0.04$ & $0.13 \pm 0.03$ & \\
\hline
\end{tabular}

Results are presented as mean \pm standard deviation. ${ }^{*} \mathrm{P}<0.05$, ${ }^{* *} \mathrm{P}<0.01,{ }^{* \star *} \mathrm{P}<0.001$ compared with control group at the same time point. EAM, experimental autoimmune myocarditis. EDV, end-diastolic volume; EF, ejection fraction; ESV, end-systolic volume; LV, left ventricle; SV, stroke volume.

Table 2 Comparison of strain parameters in the EAM group and control group

\begin{tabular}{|c|c|c|c|c|c|}
\hline Time point & Group & \multicolumn{4}{|c|}{ Strain (\%) } \\
\hline \multirow[t]{3}{*}{ Day 14} & EAM & $47.45 \pm 3.53$ & $-23.15 \pm 0.99$ & $45.50 \pm 4.51$ & $-21.96 \pm 0.99$ \\
\hline & Control & $49.00 \pm 4.80$ & $-23.89 \pm 1.12$ & $48.36 \pm 6.32$ & $-23.05 \pm 1.54$ \\
\hline & $P$ value & 0.32 & 0.065 & 0.17 & $0.028^{*}$ \\
\hline \multirow{2}{*}{ Day 21} & Control & $48.33 \pm 5.86$ & $-23.67 \pm 1.57$ & $48.48 \pm 6.50$ & $-23.18 \pm 1.74$ \\
\hline & $P$ value & $0.000^{*}$ & $0.000^{*}$ & $0.000^{*}$ & $0.000^{*}$ \\
\hline
\end{tabular}

Results are presented as mean \pm standard deviation. *indicates statistical significance $(\mathrm{P}<0.05)$. EcC ${ }_{\mathrm{SAX}}$, circumferential strain; Ell ${ }_{\mathrm{LAX}}$, longitudinal strain; Err LAX $_{\text {, radial strain from long axis; Err }}$ SAX, radial strain from short axis.

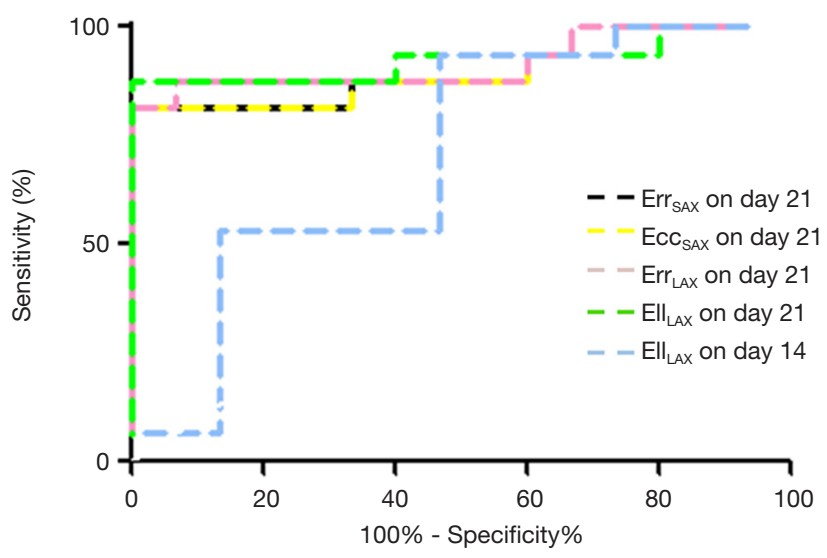

Figure 4 Receiver operating characteristic curves for left ventricle strain parameters for differentiating the control from myocarditis on days 21 and 14. Myocardial strain analysis showed good diagnostic value for both early myocarditis and acute myocarditis. Ecc $\mathrm{SAX}_{\mathrm{XX}}$, circumferential strain; Ell $\mathrm{LAX}_{\mathrm{LX}}$, longitudinal strain; Err $_{\mathrm{LAX}}$, radial strain from long axis; Err $_{\mathrm{SAX}}$, radial strain from short axis. 
Table 3 Diagnostic performance of different cardiac magnetic resonance strain parameters for the diagnosis of early and acute myocarditis

\begin{tabular}{lcccccc}
\hline & Strain & Sensitivity (\%) & Specificity (\%) & AUC (\%) & PPV (\%) & NPV (\%) \\
\hline Early myocarditis & $\operatorname{Ell}_{\text {LAX }}(>-23.41 \%)$ & $93[68-100]$ & $53[27-79]$ & $70[51-85]$ & $67[44-85]$ & $89[49-100]$ \\
Acute myocarditis & $\operatorname{Err}_{\text {SAX }}(<39.33 \%)$ & $81[54-96]$ & $100[78-100]$ & $90[74-98]$ & $100[74-100]$ & $83[59-96]$ \\
& $\operatorname{Ecc}_{\text {SAX }}(>-22.21 \%)$ & $81[54-96]$ & $100[78-100]$ & $89[73-97]$ & $100[74-100]$ & $83[59-96]$ \\
& $\operatorname{Err}_{\text {LAX }}(<38.57 \%)$ & $81[54-96]$ & $100[78-100]$ & $92[76-99]$ & $100[74-100]$ & $83[59-96]$ \\
& $\operatorname{Ell}_{\text {LAX }}(>-21.12 \%)$ & $88[62-98]$ & $100[78-100]$ & $93[77-99]$ & $100[77-100]$ & $88[64-99]$ \\
\hline
\end{tabular}

Results are presented as mean \pm standard deviation. AUC, area under the curve; ECC $_{S A x}$, circumferential strain; Ell ${ }_{\perp A x}$, longitudinal strain; Err $_{\text {LAX }}$, radial strain from long axis; Err $_{S A X}$, radial strain from short axis; NPV, negative predictive value; PPV, positive predictive value.

cardiac function in myocarditis. In a prospective study, suspected acute myocarditis patients showed reduced radial strain (RS), circumferential strain (CS), and longitudinal strain (LS) when compared with controls (RS: $28.9 \% \pm 8.5 \%$ vs. $32.4 \% \pm 7.4 \%$, CS: $-23.0 \% \pm 5.8 \%$ vs. $-27.4 \% \pm 3.4 \%$, LS: $-19.5 \% \pm 4.4 \%$ vs. $-23.6 \% \pm 3.1 \%$; $\mathrm{P}<0.05)$. Furthermore, the results of the present study found that myocardial strain was correlated with edema (28). Consistent with previous studies, a significant decrease in all strain parameters was also demonstrated in our acute myocarditis rats $(20,28,29)$. These phenomena may be ascribed to myocardial fiber orientation, where epicardial fibers, obliquely oriented, contributed to Ell ${ }_{\mathrm{LAX}}$ and $\mathrm{Ecc}_{\mathrm{SAX}}$, while mid-myocardial fibers were circumferentially oriented and contributed to Ecc $_{\mathrm{SAX}}$ and full myocardial layer-derived radial strain (29-31). In early myocarditis, we found that only myocardial epicardial damage, and therefore longitudinal strain, tended to decrease, whereas circumferential strain and radial strain were less affected. In acute myocarditis, the lesion predominately involved the epicardium and midwall, and even the full layer. These impaired fibers can potentially be detected by an abnormal longitudinal strain and circumferential strain, and our study further validated this finding. However, unlike previous studies, we found that intergroup differences in radial strain parameters were also extremely pronounced in acute myocarditis, which could be due to three reasons (28). First, in the EAM group, most of the acute myocarditis rats developed significant epicardial, mid-myocardial, and even full-layer, myocardial inflammation, whereas patients with acute myocarditis most commonly have epicardial damage $(2,28)$. Therefore, myocardial lesions in rats may be even worse than those of patients. The radial strain derived from fulllayer myocardial deformation, whereas almost full-layer myocardial inflammation occurred in the acute myocarditis rats $(30,31)$. Therefore, the degree of radial strain reduction unexpectedly exceeded that of patients. Second, epicardial effusion probably restrained the radial mechanic motion. Third, previous studies reported that radial strain is less reproductive and more variable, thus resulting in different results (32-34). Although the EF of acute myocarditis was also reduced, myocardial strain can better explain the mechanism of impaired cardiac function.

Our study revealed that all the global strain parameters, especially $E \mathrm{El}_{\mathrm{LAX}}$, demonstrated excellent diagnostic value (all AUC $>0.9$ ) for detecting acute myocarditis, thus suggesting that myocardial strain analyses could provide additional and important diagnostic information for the assessment of acute myocarditis. Compared with a previous study, our study had a higher sensitivity and specificity to all the global strain parameters, which may be explained by the fact that the severity of our acute myocarditis model was worse than that of patients with acute myocarditis (28). This could be because day 21 after the immunization was the most dangerous period for the rats, whereas clinical patients with acute myocarditis may vary in the severity of their cardiac symptoms, contributing little to the overlap of strain parameters between the myocarditis rats and the control rats (12).

Based on previous studies and our results, day 14 could be considered the early stage of myocarditis, and day 21 as the acute stage of myocarditis $(11,12,24)$. Histologically and immunohistochemically, myocardial pathological severity and macrophage infiltration began to increase 14 days after immunization and peaked at 21 days. The strain results corresponded to the histological findings in the present study. Only the longitudinal strain decreased at 14 days; thereafter, all strain parameters were significantly reduced at day 21 . The process of myocardial strain value was consistent with the process of myocardial inflammation throughout myocarditis. Myocardial epicardial edema, 
necrosis, and inflammatory infiltration contributed to the decrease of the longitudinal strain at day 14 after immunization. Subsequently, the increase of inflammatory infiltration and destruction aggravated myocyte necrosis, rarefaction, and fibrosis development, which impaired the normal structure that sustained the normal cardiac mechanic function, resulting in myocardial strain decrease in all directions at day 21. Overall, these results suggest that TT strain analysis could not only directly assess cardiac dysfunction but also indirectly reflect the severity of myocardial tissue damage in the early and acute myocarditis rats, which may prove to be a promising non-invasive imaging diagnostic indicator for the evaluation of cardiac involvement in myocarditis.

\section{Limitations}

The main limitations of the current study were that it did not use the LLC for evaluating myocarditis, because both $T_{2}-$ and $\mathrm{T}_{1}$-weighted imaging sequences of the 7.0 $\mathrm{T}$ preclinical CMR are complex in animals, and the long scanning duration could increase the mortality of rats. In addition, the quick heart rate caused substantial artifacts and poor image quality. Second, we only measured global strain parameters but did not measure segmental strain parameters, and minor focal lesions might have been overlooked as a result. Therefore, segmental strain analysis should be further investigated. The segmental strain was less reproducible and had some limitations, and reliable software has yet to be developed and optimized (32). The results of the present study should be substantiated in future studies.

\section{Conclusions}

CMR-TT-derived myocardial strain analysis can be used to differentiate early myocarditis from normal cases, and has excellent diagnostic performance in detecting acute myocarditis and reliably evaluating the different stages of the disease in a preclinical model. Such translational myocarditis research in animal studies can provide opportunities for further clinical studies. CMR-TT strain analysis could evaluate tissue injuries non-invasively and has the potential to facilitate understanding of the disorder to develop novel therapies.

\section{Acknowledgments}

Funding: The present study was supported by the National
Natural Science Foundation of China (no. 8193000682, 81520108014,81771800 , and 81829003), the State Key Project of Research and Development Plan of China (no. 2016YFA0201402), and the International Cooperation Project of Science and Technology Plan of Sichuan (no. 2017HH0045). The datasets used and/or analyzed during the current study are available from the corresponding author upon request.

\section{Footnote}

Conflicts of Interest: All authors have completed the ICMJE uniform disclosure form (available at http://dx.doi. org/10.21037/qims-20-122). The authors have no conflicts of interest to declare.

Ethical Statement: All procedures were performed under an approved protocol by the Institutional Animal Care and Use Committee of Animal Experiment Center, West China Hospital, Sichuan University. The rats were maintained in accordance with the requirements of the National Institutes of Health Guide for the Care and Use of Laboratory Animal and the Association for Assessment and Accreditation of Laboratory Animal Care. All experimental protocols were reviewed and approved by the Experimental Animal Ethics Committee of West China Hospital, Sichuan University, China.

Open Access Statement: This is an Open Access article distributed in accordance with the Creative Commons Attribution-NonCommercial-NoDerivs 4.0 International License (CC BY-NC-ND 4.0), which permits the noncommercial replication and distribution of the article with the strict proviso that no changes or edits are made and the original work is properly cited (including links to both the formal publication through the relevant DOI and the license). See: https://creativecommons.org/licenses/by-nc-nd/4.0/.

\section{References}

1. Aretz HT, Billingham ME, Edwards WD, Factor SM, Fallon JT, Fenoglio JJ Jr, Olsen EG, Schoen FJ. Myocarditis. A histopathologic definition and classification. Am J Cardiovasc Pathol 1987;1:3-14.

2. Friedrich MG, Sechtem U, Schulz-Menger J, Holmvang G, Alakija P, Cooper LT, White JA, Abdel-Aty H, Gutberlet M, Prasad S, Aletras A, Laissy JP, Paterson I, Filipchuk NG, Kumar A, Pauschinger M, Liu P; 
International Consensus Group on Cardiovascular

Magnetic Resonance in Myocarditis. Cardiovascular magnetic resonance in myocarditis: A JACC White Paper. J Am Coll Cardiol 2009;53:1475-87.

3. Cooper LT Jr. Myocarditis. N Engl J Med 2009;360:1526-38.

4. Caforio AL, Pankuweit S, Arbustini E, Basso C, GimenoBlanes J, Felix SB, Fu M, Heliö T, Heymans S, Jahns R, Klingel K, Linhart A, Maisch B, McKenna W, Mogensen J, Pinto YM, Ristic A, Schultheiss HP, Seggewiss H, Tavazzi L, Thiene G, Yilmaz A, Charron P, Elliott PM; European Society of Cardiology Working Group on Myocardial and Pericardial Diseases. Current state of knowledge on aetiology, diagnosis, management, and therapy of myocarditis: a position statement of the European Society of Cardiology Working Group on Myocardial and Pericardial Diseases. Eur Heart J 2013;34:2636-48, 2648a-2648d.

5. Ammirati E, Cipriani M, Moro C, Raineri C, Pini D, Sormani P, Mantovani R, Varrenti M, Pedrotti P, Conca C, Mafrici A, Grosu A, Briguglia D, Guglielmetto S, Perego GB, Colombo S, Caico SI, Giannattasio C, Maestroni A, Carubelli V, Metra M, Lombardi C, Campodonico J, Agostoni P, Peretto G, Scelsi L, Turco A, Di Tano G, Campana C, Belloni A, Morandi F, Mortara A, Cirò A, Senni M, Gavazzi A, Frigerio M, Oliva F, Camici PG; Registro Lombardo delle Miocarditi. Clinical Presentation and Outcome in a Contemporary Cohort of Patients With Acute Myocarditis: Multicenter Lombardy Registry. Circulation 2018;138:1088-99.

6. Yelgec NS, Dymarkowski S, Ganame J, Bogaert J. Value of MRI in patients with a clinical suspicion of acute myocarditis. Eur Radiol 2007;17:2211-7.

7. Unterberg-Buchwald C, Ritter CO, Reupke V, Wilke RN, Stadelmann C, Steinmetz M, Schuster A, Hasenfuß G, Lotz J, Uecker M. Targeted endomyocardial biopsy guided by real-time cardiovascular magnetic resonance. J Cardiovasc Magn Reson 2017;19:45.

8. Tschöpe C, Kherad B, Schultheiss HP. How to perform an endomyocardial biopsy?. Turk Kardiyol Dern Ars 2015;43:572-5.

9. Tschöpe C, Cooper LT, Torre-Amione G, Van Linthout S. Management of Myocarditis-Related Cardiomyopathy in Adults. Circ Res 2019;124:1568-83.

10. Ammirati E, Moroni F, Sormani P, Peritore A, Milazzo A, Quattrocchi G, Cipriani M, Oliva F, Giannattasio C, Frigerio M, Roghi A, Camici PG, Pedrotti P. Quantitative changes in late gadolinium enhancement at cardiac magnetic resonance in the early phase of acute myocarditis. Int J Cardiol 2017;231:216-21.

11. Korkusuz H, Esters P, Huebner F, Bug R, Ackermann H, Vogl TJ. Accuracy of cardiovascular magnetic resonance in myocarditis: comparison of MR and histological findings in an animal model. J Cardiovasc Magn Reson 2010;12:49.

12. Jeuthe S, Wassilew K, O H-Ici D, da Silva TF, Münch F, Berger F, Kuehne T, Pieske B, Messroghli DR. Myocardial T1 maps reflect histological findings in acute and chronic stages of myocarditis in a rat model. J Cardiovasc Magn Reson 2016;18:19.

13. Ferreira VM, Schulz-Menger J, Holmvang G, Kramer CM, Carbone I, Sechtem U, Kindermann I, Gutberlet M, Cooper LT, Liu P, Friedrich MG. Cardiovascular Magnetic Resonance in Nonischemic Myocardial Inflammation: Expert Recommendations. J Am Coll Cardiol 2018;72:3158-76.

14. Liu T, Wang C, Li S, Zhao Y, Li P. Age- and genderrelated normal references of right ventricular strain values by tissue tracking cardiac magnetic resonance: results from a Chinese population. Quant Imaging Med Surg 2019;9:1441-50.

15. Cui Y, Cao Y, Song J, Dong N, Kong X, Wang J, Yuan Y, Zhu X, Yan X, Greiser A, Shi H, Han P. Association between myocardial extracellular volume and strain analysis through cardiovascular magnetic resonance with histological myocardial fibrosis in patients awaiting heart transplantation. J Cardiovasc Magn Reson 2018;20:25.

16. Kovács Z, Kormányos Á, Domsik P, Kalapos A, Lengyel C, Ambrus N, Ajtay Z, Piros GÁ, Forster T, Nemes A. Left ventricular longitudinal strain is associated with mitral annular fractional area change in healthy subjectsResults from the three-dimensional speckle tracking echocardiographic MAGYAR-Healthy Study. Quant Imaging Med Surg 2019;9:304-11.

17. Kostakou PM, Kostopoulos VS, Tryfou ES, Giannaris VD, Rodis IE, Olympios CD, Kouris NT. Subclinical left ventricular dysfunction and correlation with regional strain analysis in myocarditis with normal ejection fraction. A new diagnostic criterion. Int J Cardiol 2018;259:116-21.

18. Mordi I, Bezerra H, Carrick D, Tzemos N. The Combined Incremental Prognostic Value of LVEF, Late Gadolinium Enhancement, and Global Circumferential Strain Assessed by CMR. JACC Cardiovasc Imaging 2015;8:540-9.

19. Baeßler B, Treutlein M, Schaarschmidt F, Stehning C, Schnackenburg B, Michels G, Maintz D, Bunck AC. A novel multiparametric imaging approach to acute 
myocarditis using T2-mapping and CMR feature tracking. J Cardiovasc Magn Reson 2017;19:71.

20. Baeßler B, Schaarschmidt F, Dick A, Michels G, Maintz D, Bunck AC. Diagnostic implications of magnetic resonance feature tracking derived myocardial strain parameters in acute myocarditis. Eur J Radiol 2016;85:218-27.

21. Kodama M, Matsumoto Y, Fujiwara M, Masani F, Izumi

T, Shibata A. A novel experimental model of giant cell myocarditis induced in rats by immunization with cardiac myosin fraction. Clin Immunol Immunopathol 1990;57:250-62.

22. Veeraveedu PT, Watanabe K, Ma M, Palaniyandi SS, Yamaguchi K, Suzuki K, Kodama M, Aizawa Y. Torasemide, a long-acting loop diuretic, reduces the progression of myocarditis to dilated cardiomyopathy. Eur J Pharmacol 2008;581:121-31.

23. Kodama M, Matsumoto Y, Fujiwara M, Zhang SS, Hanawa H, Itoh E, Tsuda T, Izumi T, Shibata A. Characteristics of giant cells and factors related to the formation of giant cells in myocarditis. Circ Res 1991;69:1042-50.

24. Rinkevich-Shop S, Konen E, Kushnir T, Epstein FH, Landa-Rouben N, Goitein O, Ben Mordechai T, Feinberg MS, Afek A, Leor J. Non-invasive assessment of experimental autoimmune myocarditis in rats using a 3 T clinical MRI scanner. Eur Heart J Cardiovasc Imaging 2013;14:1069-79.

25. Bondarenko O, Beek AM, Hofman MB, Kühl HP, Twisk JW, van Dockum WG, Visser CA, van Rossum AC. Standardizing the definition of hyperenhancement in the quantitative assessment of infarct size and myocardial viability using delayed contrast-enhanced CMR. J Cardiovasc Magn Reson 2005;7:481-5.

26. Gao P, Yee R, Gula L, Krahn AD, Skanes A, LeongSit P, Klein GJ, Stirrat J, Fine N, Pallaveshi L, Wisenberg G, Thompson TR, Prato F, Drangova M, White JA. Prediction of arrhythmic events in ischemic and dilated cardiomyopathy patients referred for implantable cardiac defibrillator: evaluation of multiple scar quantification measures for late gadolinium enhancement magnetic resonance imaging. Circ

Cite this article as: Zhu J, Chen Y, Xu Z, Wang S, Wang L, Liu X, Gao F. Non-invasive assessment of early and acute myocarditis in a rat model using cardiac magnetic resonance tissue tracking analysis of myocardial strain. Quant Imaging Med Surg 2020;10(11):2157-2167. doi: 10.21037/qims-20-122
Cardiovasc Imaging 2012;5:448-56.

27. Korkusuz H, Esters P, Naguib N, Nour Eldin NE, Lindemayr S, Huebner F, Koujan A, Bug R, Ackermann H, Vogl TJ. Acute myocarditis in a rat model: late gadolinium enhancement with histopathological correlation. Eur Radiol 2009;19:2672-8.

28. Luetkens JA, Schlesinger-Irsch U, Kuetting DL, Dabir D, Homsi R, Doerner J, Schmeel FC, Fimmers R, Sprinkart AM, Naehle CP, Schild HH, Thomas D. Feature-tracking myocardial strain analysis in acute myocarditis: diagnostic value and association with myocardial oedema. Eur Radiol 2017;27:4661-71.

29. Doerner J, Bunck AC, Michels G, Maintz D, Baeßler B. Incremental value of cardiovascular magnetic resonance feature tracking derived atrial and ventricular strain parameters in a comprehensive approach for the diagnosis of acute myocarditis. Eur J Radiol 2018;104:120-8.

30. Sengupta PP, Tajik AJ, Chandrasekaran K, Khandheria $\mathrm{BK}$. Twist mechanics of the left ventricle: principles and application. JACC Cardiovasc Imaging 2008;1:366-76.

31. Ho SY. Anatomy and myoarchitecture of the left ventricular wall in normal and in disease. Eur J Echocardiogr 2009;10:iii3-iii7.

32. Schmidt B, Dick A, Treutlein M, Schiller P, Bunck AC, Maintz D, Baeßler B. Intra- and inter-observer reproducibility of global and regional magnetic resonance feature tracking derived strain parameters of the left and right ventricle. Eur J Radiol 2017;89:97-105.

33. Taylor RJ, Moody WE, Umar F, Edwards NC, Taylor TJ, Stegemann B, Townend JN, Hor KN, Steeds RP, Mazur W, Leyva F. Myocardial strain measurement with featuretracking cardiovascular magnetic resonance: normal values. Eur Heart J Cardiovasc Imaging 2015;16:871-81.

34. Andre F, Steen H, Matheis P, Westkott M, Breuninger K, Sander Y, Kammerer R, Galuschky C, Giannitsis E, Korosoglou G, Katus HA, Buss SJ. Age- and genderrelated normal left ventricular deformation assessed by cardiovascular magnetic resonance feature tracking. J Cardiovasc Magn Reson 2015;17:25. 